\title{
Experimental determination of the band gap dependence of Auger recombination in InGaAs/InP multiple quantum well lasers at room temperature
}

\author{
N. F. Massé, A. R. Adams, and S. J. Sweeney ${ }^{a)}$ \\ Advanced Technology Institute, University of Surrey, Guildford, Surrey GU2 7XH, United Kingdom
}

(Received 11 January 2007; accepted 5 March 2007; published online 17 April 2007)

\begin{abstract}
The band gap dependencies of the threshold current and its radiative component are measured using high pressure techniques. Detailed theoretical calculations show that the band gap dependence of the internal losses plays a significant role in the band gap dependence of the radiative current. Temperature dependence measurements show that the radiative current accounts for $20 \%$ of the total threshold current at room temperature. This allows us to determine the pressure dependence of the non-radiative Auger recombination current, and hence to experimentally obtain the variation of the Auger coefficient $C$ with band gap. (C) 2007 American Institute of Physics.
\end{abstract}

[DOI: 10.1063/1.2722041]

The relative importance of different carrier recombination processes occurring in semiconductor diode lasers is strongly dependent on the band structure, material quality and laser geometry (e.g., cavity length, facet coatings, etc.). While a small amount of radiative recombination via spontaneous emission is desirable (to seed the stimulated emission process), other nonradiative recombination paths may contribute to the laser threshold current and are detrimental to the performance of the devices. For $\operatorname{InGaAs}(\mathrm{P}) / \mathrm{InP}$ devices operating over the telecommunications range $(1.3-1.6 \mu \mathrm{m})$, it is known that Auger recombination plays an important role and is responsible for their high temperature sensitivity ${ }^{1-4}$. In order to understand these limiting processes and consequently to improve the performance of the devices, it is necessary to separate the contribution of the different recombination current paths. ${ }^{1-6}$ Hydrostatic pressure is an ideal tool to investigate the current paths and their variation with band gap, and hence operating wavelength. In long wavelength semiconductor multiple quantum well lasers where leakage currents are small, it has been shown ${ }^{1,5}$ that the threshold current $I_{\text {th }}$ can be written as the sum of monomolecular $(\propto n)$, radiative $\left(\propto n^{2}\right)$, and Auger $\left(\propto n^{3}\right)$ recombination currents, where $n$ is the carrier density at threshold (assuming that the hole and electron densities are equal). Thus $I_{\mathrm{th}}=e V\left(A n+B n^{2}+C n^{3}\right)$ where $e$ is the electronic charge, $V$ is the volume of the active region, and $A, B$, and $C$ are the monomolecular, radiative, and Auger recombination coefficients, respectively. Good growth quality means that the monomolecular current is negligible in the InGaAs devices studied here. ${ }^{1,5}$ The relative magnitudes of the remaining radiative and nonradiative currents have been experimentally studied via temperature and high pressure measurements. ${ }^{1,5}$ Previous analyses were based on the theoretical assumption that for an ideal quantum well laser, the radiative current is proportional to the square of the band gap: $I_{\text {rad }} \propto E_{g}^{2}$, where $E_{g}$ is the band gap ${ }^{7}$ although this had not been experimentally verified at room temperature. The pressure dependence of the radiative current has already been verified at low temperatures using a helium gas pressure

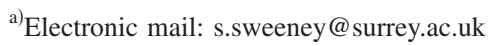

system. ${ }^{8}$ Since at cryogenic temperatures $(T<120 \mathrm{~K})$ all of the injected carriers are involved in radiative recombination, $I_{\text {rad }}$ is obtained directly by measuring the threshold current. In the work presented here, the band gap dependence of the radiative current is experimentally studied at room temperature where nonradiative recombination also occurs. In this experiment, the radiative current is determined by collecting the spontaneous emission emitted from a window milled in the $n$-side substrate of the laser using an optical fiber. This ensures that one is measuring pure spontaneous emission independent of the effects of gain and loss along the cavity. ${ }^{5}$ The spontaneous emission is analyzed using an optical spectrum analyzer to ensure that we are collecting pure spontaneous emission (as evident from the shape of the spectra). The amount of spontaneously emitted light is determined by integrating the spectra at each current. The variation of the radiative current at threshold is determined from the pinning level of the integrated spontaneous emission. ${ }^{5}$ The facet emission light-current characteristic was also measured with a broad area germanium detector. The devices were positioned in a spring clip in a piston-in-cylinder high pressure system as described elsewhere. ${ }^{9}$ The lasers were driven by a pulsed source with 500-ns-long pulses at a repetition rate of $10 \mathrm{kHz}$ to avoid internal heating. A set of 1.5-mm-long ascleaved InGaAs/InP laser diodes emitting at $1.5 \mu \mathrm{m}$ was investigated. Their active region consists of four InGaAs $0.6 \%$ compressively strained quantum wells surrounded by unstrained InGaAsP barriers. The 100- $\mu \mathrm{m}$-diameter circular substrate windows were milled by a Focused Ion Beam. The threshold current densities of the devices $\left(\sim 900 \mathrm{~A} \mathrm{~cm}^{-2}\right)$, measured before and after milling, were the same within experimental error and hence we are confident that the milling did not influence the lasing properties.

The results of the pressure measurements are shown in Fig. 1. The threshold current $I_{\text {th }}$ decreases by $\sim 23 \%$ over a range of $8 \mathrm{kbars}$ (shown by the full squares), while $I_{\text {rad }}$ at threshold increases by $\sim 10 \%$ (open circles). Also shown is the expected band gap dependence of the radiative current as predicted by simple theory, for which $I_{\text {rad }} \propto E_{g}^{2}$. It can be seen that the measured radiative current increases less than the simple $E_{g}^{2}$ model. To replace this simple model, a more rigorous calculation of the radiative current was undertaken. 


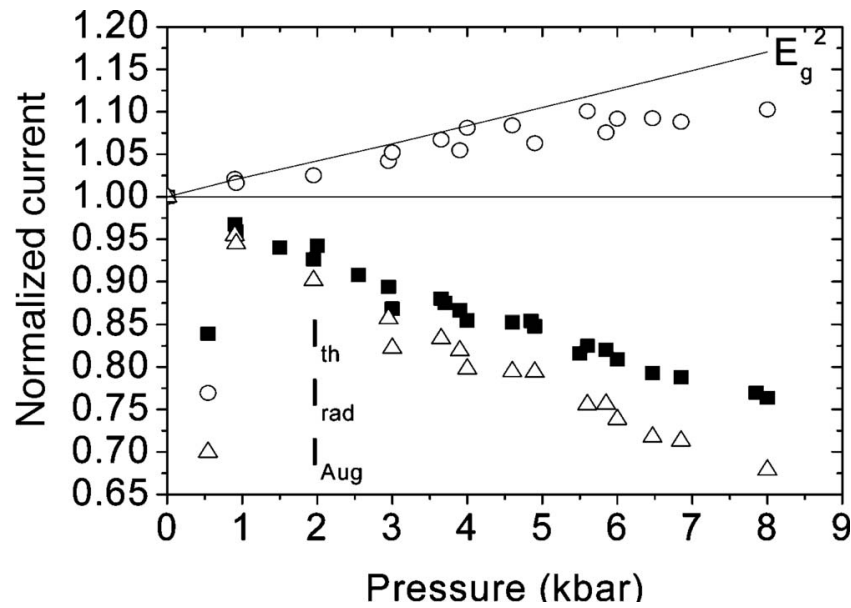

FIG. 1. Experimental variation of the threshold radiative and nonradiative currents at threshold as a function of pressure. The solid line shows the variation of $E_{g}^{2}$.

To calculate $I_{\text {rad }}$ as a function of pressure, a six-band $k \cdot p$ Hamiltonian was used to determine the quantum well $(\mathrm{QW})$ band structure, taking into account the changes in the effective masses and band gaps as a function of pressure. Poisson's and Schrödinger's equations were solved selfconsistently in the calculation of both the conduction and valence bands under injection. A five-point difference method was used to account for carrier spill-over into the barrier and separate-confinement regions. The spontaneous emission rate, gain, and radiative current density were calculated from these band structures for single QWs using the density matrix formulation including Lorentzian-type broadening. Further details of the modeling can be found in Ref. 10. As pressure is applied to the lasers, the wavelength decreases by $\sim 114 \mathrm{~nm}$ over 8 kbars. This leads to a narrowing of the optical field while the dimensions of the active region remain approximately constant. The corresponding pressureinduced variation of the optical confinement factor $\Gamma$ (the overlap of the optical field with the quantum wells) was accounted for using the effective index method. ${ }^{10}$ We find that when all these changes with pressure are included the agreement with the experimental $I_{\mathrm{rad}}(P)$ is poor (dashed line in Fig. 2), suggesting that yet another factor should be taken into account. In earlier work, we showed that the internal loss at room temperature and atmospheric pressure $\alpha_{0}$ of these $1.5 \mu \mathrm{m}$ lasers is $10 \mathrm{~cm}^{-1}$. Including this as a pressure

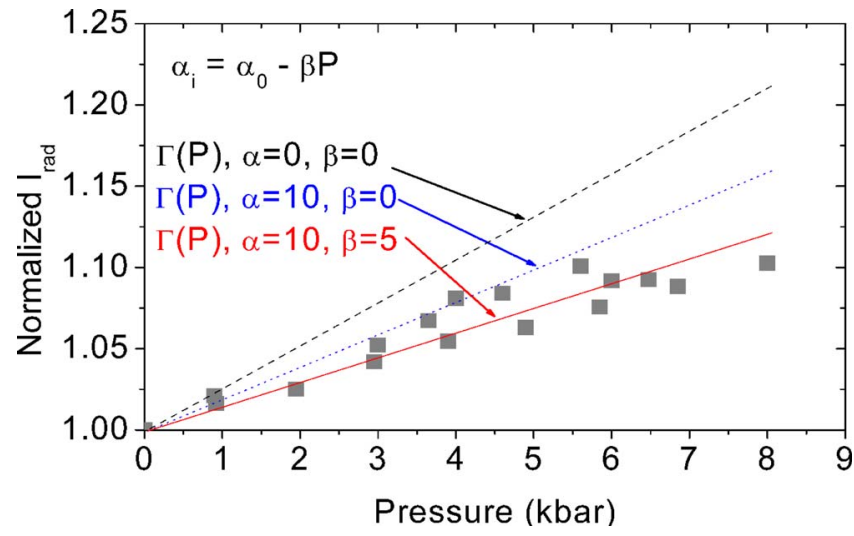

FIG. 2. (Color online) Comparison between normalized experimental data

the total pumped volume of the quantum wells). At atmo-
and the calculated radiative current.

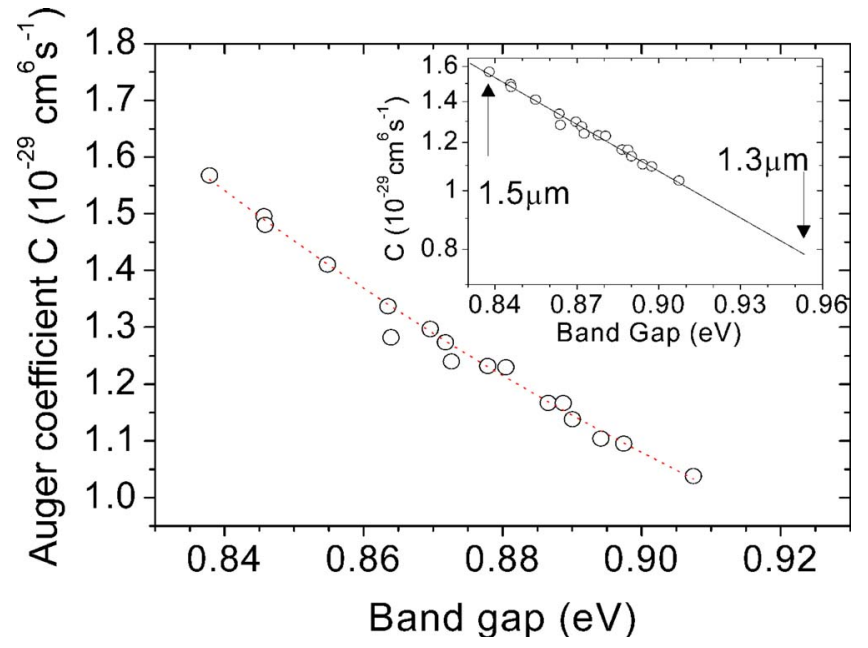

FIG. 3. (Color online) Band gap dependence of the Auger recombination coefficient $C$. The inset shows the extrapolated value of $C$ to wider band gaps (note the logarithmic scale).

independent loss results in the dotted line in Fig. 2. While this offers better agreement it still overestimates the rate of increase of radiative current with pressure. The origin of the internal loss in lasers operating at these wavelengths is due to intervalence band absorption ${ }^{11}$ whereby a photon is absorbed by an electron in the spin-orbit band which is excited to the highest (heavy hole) valence band. While the photon energy increases with pressure, the spin orbit splitting remains relatively constant. ${ }^{12}$ Thus, with increasing pressure, in order to satisfy the conservation of energy, the transitions involved in intervalence band absorption move away from the Brillouin zone center where the hole occupation becomes less, therefore reducing the intervalence band absorption rate. To take this into account, to first approximation we assume that the intervalence band absorption loss $\alpha_{i}$ varies linearly with pressure as $\alpha_{i}=\alpha_{0}-\beta P$, where $P$ is the pressure in kilobars and $\beta$ is a constant equal to the rate of change in loss with pressure. Over the studied pressure range, we find best agreement with the experimental data for $\beta$ $=0.5 \mathrm{~cm}^{-1} \mathrm{kbar}^{-1}$, as shown by the solid line in Fig. 2. This therefore suggests that the intervalence band absorption loss reduces by $\sim 40 \%$ over 8 kbars.

The fact that the radiative current increases with pressure while the threshold current decreases with pressure confirms that there is a strong nonradiative Auger current path present which decreases with increasing pressure. Temperature measurements show that $I_{\text {rad }}$ forms $20 \%$ of the threshold current at room temperature in these devices with the remaining $80 \%$ due to Auger recombination. ${ }^{1,2,5}$ This result together with our pressure dependence data allows us to experimentally determine the band gap dependence of the Auger current $I_{\text {Aug }}$ (Fig. 1). It is shown in Ref. 13 that the Auger process known as CHSH (where the energy of a Conduction band electron recombining with a hole in the Heavy hole band is given to a third hole in the Heavy hole band which is excited into the Spin split-off band) dominates in these devices. We may therefore calculate the pressure (band gap) variation of the Auger coefficient given by $C=I_{\text {Aug }} / e V p_{\text {th }}^{2} n_{\text {th }}$ for the CHSH Auger process using the calculated pressure dependencies of the electron and hole densities at threshold $\left(n_{\text {th }}\right.$ and $p_{\text {th }}$, respectively). This is shown in Fig. 3 (where $V$ is taken to be the total pumped volume of the quantum wells). At atmo- 
spheric pressure, $C$ is found to be $\sim 1.57 \times 10^{-29} \mathrm{~cm}^{6} \mathrm{~s}^{-1}$, decreasing exponentially with increasing band gap. Based on this, $C$ can be extrapolated to wider band gaps (inset, Fig. 3), where, for example, it is found to be $50 \%$ less at $E_{g}$ $=954 \mathrm{meV}(\lambda=1.3 \mu \mathrm{m})$ compared to $C$ at $1.5 \mu \mathrm{m}$. Our values are consistent with typical values reported in the literature $^{14}$ and with measurements on $1.3 \mu \mathrm{m}$ lasers for which $I_{\mathrm{Aug}} \sim 50 \% I_{\text {th }}$ at room temperature and atmospheric pressure.

To conclude, we have experimentally measured the pressure dependence of the radiative current in $1.5 \mu \mathrm{m}$ InGaAs/InP quantum well laser diodes. We find that the simple model given by $I_{\mathrm{rad}} \propto E_{g}^{2}$ is insufficient to describe the experimental data. However, good agreement was found using a full band-structure calculation if the pressure dependence of the optical confinement factor and internal losses is taken into account. Our calculations show that the decrease in the internal losses due to reduced intervalence band absorption plays a significant role in reducing the pressure dependence of the radiative current. Based on our measurements we are able to experimentally determine the band gap dependence of the Auger current and the Auger coefficient $C$.

The authors would like to thank Igor Marko for assistance with the experiments.
${ }^{1}$ S. J. Sweeney, D. McConville, N. F. Massé, R.-X. Bouyssou, A. R. Adams, C. N. Ahmad, and C. Hanke, Phys. Status Solidi B 241, 391 (2004).

${ }^{2}$ S. J. Sweeney, A. F. Phillips, A. R. Adams, E. P. O'Reilly, and P. J. A. Thijs, IEEE Photonics Technol. Lett. 10, 1076 (1998).

${ }^{3}$ P. J. A. Thiis, L. F. Tiemeijer, J. J. M. Binsma, and T. Van Dongen, IEEE J. Quantum Electron. 30, 477 (1994).

${ }^{4}$ E. P. O'Reilly and M. Silver, Appl. Phys. Lett. 63, 3318 (1993).

${ }^{5}$ A. F. Philips, S. J. Sweeney, A. R. Adams, and P. J. A. Thijs, IEEE J. Sel. Top. Quantum Electron. 5, 401 (1999).

${ }^{6}$ D. Patel, A. R. Adams, P. D. Greene, and G. D. Henshall, Electron. Lett. 18, 527 (1982).

${ }^{7}$ A. R. Adams, M. Silver, and J. Allam, in Semiconductors and Semimetals, edited by R. K. Willardson and E. R. Zeber, 310 (Academic, New York, 1998), Vol. 55, pp. 310-311.

${ }^{8}$ S. R. Jin, S. J. Sweeney, C. N. Ahmad, A. R. Adams, and B. N. Murdin, Appl. Phys. Lett. 85, 357 (2004).

${ }^{9}$ S. R. Jin, S. J. Sweeney, S. Tomic, and A. R. Adams, Appl. Phys. Lett. 82, 2335 (2003)

${ }^{10}$ M. Silver and E. P. O’Reilly, IEEE J. Quantum Electron. 311193 (1995).

${ }^{11}$ A. R. Adams, M. Asada, Y. Suematsu, and S. Arai, Jpn. J. Appl. Phys. 19, L621 (1980)

${ }^{12}$ M. Balkanski, Handbooks on Semiconductor, Optical Properties of Solids Vol. 2 (North Holland, Amsterdam, 1980), p. 197.

${ }^{13}$ S. J. Sweeney, A. R. Adams, M. silver, E. P. O'Reilly, J. R. Watling, A. B. Walker, and P. J. A Thijs, Phys. Status Solidi B 211, 525 (1999).

${ }^{14}$ G. P. Agrawal and N. K. Dutta, Long Wavelength Semiconductor Lasers (van Nostrand Reinhold, New York, 1986), p. 122. 